\title{
Zur Kenntniss des Phosphors im Muskel.
}

\author{
Von
}

J. J. R. Macleod M. B.,

aus Aberdeen, Scotland.

(Aus dem chemischen Laboratorium des physiologischen Instituts der Universität Leipzig.)

(Der Redaction zugegangen am 28. September 1899.)

Dass die von den Nieren ausgeschiedenen Phosphate durch Muskelthätigkeit zunehmen, ist durch zahlreiche Arbeiten, besonders von Engelmann, ${ }^{1}$ ) Presyz ${ }^{2}$ ) Klug und Olsavszky ${ }^{3}$ ) u. s. w. dargethan worden.

Es ist nicht unwahrscheinlich, dass diese Zunahme von dem Abbaue phosphorhaltiger Substanzen in den Muskeln herrührt, aber Versuche, welche sich mit der Art und dem Material dieses Abbaues eingehender beschäftigen, sind nur in geringer Anzahl vorhanden, und auch diese sind durchaus nicht überzeugend.

Weyl und Zeitler ${ }^{4}$ zeigten zwar, dass eine Zunahme der anorganischen Phosphate in tetanisirten Muskeln stattfindet, aber sie konnten keine Erklärung für diese Zunahme finden, sondern vermutheten nur, dass, weil die Zunahme nicht von einer Zerstörung des Lecithins abhängt, das Nuclein die organische Substanz sein müsse, von welcher die anorganischen Phosphate stammen; indessen kann dies keineswegs die $\mathrm{Zu}$ -

1) Du Bois-Reymond's Arch. 1871. S. 14 .

2) Ungarisches Arch. f. Medicin. Bd. I. S. 38.

3) Arch. f. d. ges. Physiol. Bd. LIV. S. 21.

4) Zeitschr. f. physiol. Chemie, Bd. VI. S. 557. 
nahme der anorganischen Phosphate erklären, denn die Muskeln enthalten nur eine sehr geringe Menge von Nucleoproteid. ${ }^{1}$ )

Die angewandte Methode war auch keineswegs eine einwandsfreie, und zwar aus mehrfachen Gründen. Um das Lecithin zu extrahiren, wandten W eyl und Zeitler nur kalten Alkohol und Aether an, und es ist durch Liebermann ${ }^{2}$ ) gezeigt worden, dass selbst durch Kochen mit diesen Reagentien nur ein Theil des Lecithins ausgezogen wird. Um die anorganischen Phosphate zu extrahiren, behandelten sie die lecithinfreien Rückstände mit kochendem Wasser mehrere Male je fünf Minuten lang. Dieses Verfahren kann aber, wie Kossel ${ }^{3}$ ) und Miescher gezeigt haben, eine Zerstörung von Nuclein und somit eine incorrecte Vermehrung des anorganischen Phosphors zur Folge haben.

Ausser dem Nuclein gibt es aber noch andere organische phosphorhaltige Substanzen im Muskel, welche zu der Zeit, als Weyl und Zeitler ihre Arbeit machten, noch unbekannt waren, und aus deren Zersetzung sich die Vermehrung der anorganischen Phosphate im arbeitenden Muskel erklären lassen könnte. Eine solche Substanz ist das von Siegfried entdeckte Nucleon. Thatsächlich hat Siegfried ${ }^{4}$ ) auch gefunden, dass in dem wässerigen Extract tetanisirter Muskeln sich weniger Nucleonstickstoff findet, als in demjenigen von ruhenden.

Auf den Vorschlag des Herrn Prof. Siegfried habe ich nun Versuche darüber angestellt, ob ebenso wie der Stickstoff auch der Phosphor des Nucleonmoleküls bei der Muskelarbeit abgespalten wird. Daraus liesse sich dann sowohl die Zunahme der anorganischen Phosphate im Muskelextract, als auch die Steigerung der Phosphate im Urin bei der Muskelthätigkeit wenigstens theilweise - erklären.

1) Whitfield (Journ. of Physiol. Vol. XVI, S. 487) konnte kein Nucleoalbumin im Muskel finden; und Pekelharing (diese Zeitschr. Bd. XXII. S. 245) bei einer anderen Methode nur eine recht geringe Menge.

2) Pflüger's Arch. f. Physiologie. Bd. LIV, S. ó73.

3) Zeitschr. f. physiol. Chemie, Bd. III. S. 284.

4) Zeitschr. f. physiol. Chemie, Bd. XXI. S. 376. 
Neben dem Nucleon habe ich auch den Gesammtphosphor im getrockneten Muskel und den anorganischen und organischen Phosphor in dem bei $60^{\circ} \mathrm{C}$. bereiteten wässerigen Extracte bestimmt. Bei dieser Temperatur wird zwar (bei Verwendung von vielem Wasser und dreimaligem Wiederholen dieses Extrahirens) wohl der Gesammtgehalt des Muskels an Alkaliphosphaten ausgezogen, hingegen erleiden die organischen P-haltigen Verbindungen des Fleisches dadurch sicher nicht eine wesentliche Zersetzung.

Da der Wassergehalt des Muskels sehr beträchtlich je nach dem Alter des Thieres und nach dem Fettgehalt der Muskeln, variirt, und da der Wassergehalt durch Muskelarbeit zunimmt, ${ }^{1}$ ) so wurde der Trockengehalt des Muskels bestimmt und alle Resultate auf Procente des getrockneten Muskeis berechnet.

Der Versuch wurde an Hunden ausgeführt, welche jedes Mal wenigstens vier Tage lang mit Pferdefleisch gefüttert worden waren, vor dem Versuche jedoch einige Stunden gefastet hatten. Dieselben mussten dann mehrere Stunden in einer Tretmühle, die durch freundliche Vermittelung des Herrn Prof. N. Zuntz nach dessen Angaben construirt ist, laufen, so dass das Ermüden unter völlig normalen Bedingungen erfolgte.

Um Vergleichszahlen für. die normalen Verhältnisse zu gewinnen, wurden fünf andere Hunde wenigstens zwölf Stunden lang ruhig gehalten. In beiden Fällen wurden die Hunde dann durch Verbluten getödtet. Die Muskeln der hinteren Extremitäten wurden darauf nach Möglichkeit von Fett, Nerven u. s. w. befreit und in der Fleischmaschine zerkleinert. Der so erhaltene Muskelbrei wurde gewogen und zwei kleine Portionen davon bei $100^{\circ} \mathrm{C}$. bis zum constanten Gewichte getrocknet und so die Trockensubstanz des Muskels bestimmt.

Die trockene Masse wurde dann mit Aetznatron und Salpeter in der Silberschale verschmolzen und in der Lösung

1) Hammarsten, Lehrbuch der physiol. Chemie, 1895, S. 345, und Halliburtion in Schäfer's Textbook of Physiology, p. 95, wo eine Liste der Arbeiten über diese Frage gegeben wird. 
der Schmelze der Phosphor durch Fällen mit molybdänsaurem Ammon, Lösen des gewaschenen Niederschlags in Ammoniak und Fällen mit ammoniakalischer Magnesiamixtur bestimmt. Auf diese Weise wurde somit der Gesammtphosphor des trockenen Muskels ermittelt.

Die nicht zur Trockenbestimmung verwendete Muskelmasse wurde in zwei Theile getheilt und von jedem derselben ein Extract gemacht, und zwar wurden $800 \mathrm{~g}$ Muskel zunächst $3 / 4$ Stunden lang bei einer Temperatur zwischen 50 und $60^{\circ} \mathrm{C}$. mit $1 \frac{1}{2}$ Liter Wasser unter gutern Umrühren extrahirt. Das so gewonnene Extract wurde darauf durch ein Filtertuch colirt und das ausgepresste Fleisch wiederum 3/4 Stunden lang mit 1 Liter Wasser extrahirt. Dasselbe Verfahren wurde ein drittes Mal wiederholt. Die vereinigten Extracte wurden darauf zur Coagulation des Eiweisses gekocht und nach dem Erkalten filtrirt. Das Filtrat wurde gemessen und bestimmte Volumina davon, welche 10 und $20 \mathrm{~g}$ des feuchten Muskels entsprachen, abgemessen.

Die $10 \mathrm{~g}$ Muskel entsprechende Menge wurde in einer Silberschale eingedampft, der Rückstand mit Aetznatron und Salpeter verschmolzen und der Phosphor in der oben angegebenen Weise bestimmt. Es wurde so der Gesammt-P-Gehalt des wässerigen Extractes ermittelt.

$\mathrm{Zu}$ der $20 \mathrm{~g}$ feuchten Muskels entsprechenden Menge des Extractes wurde $\mathrm{CaCl}_{2}$ in $10 \%$ iger Lösung hinzugefügt und die Lösung mit Ammoniak schwach alkalisch gemacht. Der die präformirte Phosphorsäure als Calciumphosphat enthaltende Niederschlag wurde in Salpetersäure gelöst und sein P-Gehalt abermals mittelst molybdänsauren Ammons und Magnesiamixtur ermittelt. Dies stellt uns den anorganischen Phosphor in dem wässerigen Extract dar.

Durch Abziehen des anorganischen von dem Gesammtphosphor des wässerigen Extractes erhalten wir den organischen Phosphor des wässerigen Extractes.

Es ist unwahrscheinlich, dass bei Anwendung von $\mathrm{CaCl}_{2}$ als Fällungsmittel des anorganischen Phosphors im wässerigen Extract organische P-haltige Substanzen - z. B. Inosin- 
säure $\left.{ }^{1}\right)$ - mitgefällt werden; ebenso unwahrscheinlich ist es, dass luierbei eine Zersetzung solcher organischer Phaltiger Substanzen stattfindet, und dadurch, wie dies etwa bei direkter Fällung mit Ammoniummolybdat in stark salpetersaurer Lösung in der Wärme der Fall wäre, die anorganischen Phosphate vermehrt erscheinen.

Zur Bestimmung des Gesammt-P und der anorganischen Phosphate im wässerigen Extracte waren, wie erwähnt, nur kleine Antheile desselben verwendet worden. Die Hauptmenge der Flüssigkeit diente zur Bestimmung des Nucleons. Die Eisenfällung wurde nach den Angaben von Siegfried, ${ }^{2}$ ) Ide und $\mathrm{Balke}^{3}$ ) vorgenommen. Die saure Reaction der Flüssigkeit wurde durch vorsichtigen Zusatz von Ammoniak vermindert, jedoch sorgfältig vermieden, dass die Reaction alkalisch oder neutral wurde.

In dem auf dem Wasserbade getrockneten, gepulverten und gewogenen Nucleoneisenniederschlag wurden sowohl der Stickst off - nach Kjeldahl - wie der Phosphor durch Schmelzen mit Aetznatron und Salpeter bestimmt und beides. für $100 \mathrm{~g}$ getrockneten Muskels berechnet.

\section{Versuche an normalen Hunden.}

Versuch I. Junger, $24 \mathrm{~kg}$ schwerer Jagdhund; gelegentlich einer Operation durch Verblutung aus der Vena Portae gestorben. Vorher mehrere Tage lang ruhig und während der letzten 12 Stunden nüchtern. Die Muskeln enthielten viel Fett.

\section{Wassergehalt.}

A. 10,1168 g feuchter Muskel lieferten

$2,9318 \mathrm{~g}$ Trockensubstanz.

Wassergehalt des feuchten Muskels 71,02 \% $\%$.

1) Um das Calciumsalz der Inosinsäure zu bilden, braucht man anf der einen Seite eine concentrirte Lösung von Inosinsäure und auf der anderen eine concentrirte Lösung von $\mathrm{CaCl}_{2}$, und das Salz scheidet sich nur nach längerem Stehen aus. (Haiser, Monatshefte für Chemie, 1895, S. 190.)

2) Zeitschr. f. physiol. Chemie, Bd. XXI. S. 351.

3) Zeitschr. f. physiol. Chemie, Bd. XXI. S. 380 . 
B. $10,522 \mathrm{~g}$ feuchter Muskel lieferten

$2,9870 \mathrm{~g}$ Trockensubstanz.

Wassergehalt des feuchten Muskels 71,61\%.

Mittel $=\mathbf{7 1 , 3 1} \%$.

Daher Trockensubstanz des feuchten Muskels $=\mathbf{2 8 , 6 9} \%$.

II. Gesammt-Phosphorgehalt des Muskels.

A. $2,9318 \mathrm{~g}$ getrockneten Muskels gaben

$0,0745 \mathrm{~g} \mathrm{Mg}_{2} \mathrm{P}_{2} \mathrm{O}_{i}=0,3547 \% \mathrm{P}$.

B. $2,9870 \mathrm{~g}$ getrockneten Muskels gaben

$0,0785 \mathrm{~g} \mathrm{Mg}_{2} \mathrm{P}_{2} \mathrm{O}_{7}=0,3640 \% \mathrm{P}$.

$$
\text { Mittel: } \mathbf{0 , 3 5 9} \% \text {. }
$$

\section{Wässeriger Extract.}

A. $800 \mathrm{~g}$ feuchten Muskels gaben $3880 \mathrm{ccm}$. wässerigen Extractes

1. Gesammt-P. $48,5 \mathrm{ccm}$. des wässerigen Extractes (= $10 \mathrm{~g}$ feuchten Muskels $=2,869 \mathrm{~g}$ trockenen Muskels) lieferten $0,0495 \mathrm{~g} \mathrm{Mg}_{2} \mathrm{P}_{2} \mathrm{O}_{7}$, entsprechend $0,238 \% \mathrm{P}$, bezogen auf Trockensubstanz.

2. Anorganischer P. $97 \mathrm{ccm}$. wässerigen Extractes $(=20 \mathrm{~g}$ feuchten Muskels $=\tilde{5}, 738 \mathrm{~g}$ Trockensubstanz) lieferten $0,0777 \mathrm{~g} \mathrm{Mg}_{2} \mathrm{P}_{2} \mathrm{O}_{7}$, entsprechend $0,1888 \% \mathrm{P}$, bezogen auf Trockensubstanz.

3. Organischer P (als Differenz berechnet) $=0,0492 \% \mathrm{P}$ bezogen auf Trockensubstanz.

4. Nucleoneisenniederschlag. $3734 \mathrm{ccm}$. wässerigen Extractes $(=770 \mathrm{~g}$ feuchten Muskels $=220,9$ Trockensubstanz) lieferten 6,89ら g Eisenniederschlag mit $3,41 \% \mathrm{~N}$ und $0,89 \% \mathrm{P}$.

Nucleon-N der Trockensubstanz $=0,107 \%$.

Nucleon-P » 》. $\quad=0,0278 \%$.

B. Nur Nucleon wurde bestimmt, $3878,8 \mathrm{ccm}$. wässerigen Extractes (=770 g feuchten Muskels $=220,9$ Trockensubstanz) lieferten 7,304 $\mathrm{g}$ Eisenniederschlag mit $3,6 \% \mathrm{~N}$ und $0,80 \% \mathrm{P}$.

Nucleon- $\mathrm{N}$ der Trockensubstanz $=0,119 \%$.

Nucleon-P » $>0,026 \%$.

Versuch II. Alter, 22: ${ }^{\prime} \mathrm{kg}$ schwerer Bullenbeisser, durch Verblutung aus den Carotiden getödtet. Vorher mehrere Tage lang ruhend und während der letzten 12 Stunden nüchtern. Die Muskeln waren dunkelroth gefärbt und enthielten weniger Fett als in $\mathrm{Nr}$. I.

\section{Wassergehalt.}

A. $14,4722 \mathrm{~g}$ feuchter Muskel lieferten 4,2287 g Trockensubstanz.

Wassergehalt des feuchten Muskels 70,78\% 
B. $12,5875 \mathrm{~g}$ feuchter Muskel lieferten

3,6655 g Trockensubstanz.

Wassergehalt des feuchten Muskels 70,80\%.

Mittel $=\mathbf{7 0 , 7 9} \%$.

Daher Trockensubstanz des feuchten Muskels $=\mathbf{2 9 , 2 1} \%$.

\section{Gesammt-Phosphor.}

A. 4,2287 g getrockneter Muskel gaben $0,1105 \mathrm{~g} \mathrm{Mg}_{2} \mathrm{P}_{2} \mathrm{O}_{7}=0,364 \% \mathrm{P}$.

B. $3,6655 \mathrm{~g}$ getrockneter Muskel gaben $0,93 \mathrm{~g} \mathrm{Mg}_{2} \mathrm{P}_{2} \mathrm{O}_{7}=0,360 \% \mathrm{P}$.

$$
\text { Mittel } \mathbf{0 , 3 6 2} \% \text {. }
$$

\section{Wässeriger Extract.}

A. $1040 \mathrm{~g}$ feuchten Muskels gaben $5387 \mathrm{ccm}$. wässerigen Extractes.

1. Ge s a mmt-P verloren.

2. A n org a n is c her P. $103,6 \mathrm{ccm}$. wässerigen Extractes $(=20 \mathrm{~g}$ feuchten Muskels $=5,84 \mathrm{~g}$ Trockensubstanz) lieferten 0,092 $\mathrm{g}$ $\mathrm{Mg}_{2} \mathrm{P}_{2} \mathrm{O}_{7}$, entsprechend $0,2191 \% \mathrm{P}$, bezogen auf Trockensubstanz.

3. Organischer P.

4. $\mathrm{Nucle}$ on eisenniederschlag. $5271,60 \mathrm{ccm}$. wässerigen Extractes $(=1010 \mathrm{~g}$ feuchten Muskels $=294 \mathrm{~g}$ Trockensubstanz) lieferten $8,526 \mathrm{~g}$ Eisenniederschlag mit $2,4 \% \mathrm{~N}$ und $1,21 \% \mathrm{P}$.

Nucleon-N der Trockensubstanz $0,0693 \%$.

Nucleon-P » $\quad 0,0350 \%$.

B. $1040 \mathrm{~g}$ feuchten Muskels gaben $5160 \mathrm{ccm}$. wässerigen Extractes.

1. Gres a m m t-P. $49,9 \mathrm{ccm}$. des wässerigen Extractes $(=10 \mathrm{~g}$ feuchten Muskels $=2,92 \mathrm{~g}$ Trockensubstanz) lieferten $0,0555 \mathrm{~g}$ $\mathrm{Mg}_{2} \mathrm{P}_{2} \mathrm{O}_{7}$, entsprechend $0,2671 \% \mathrm{P}$, bezogen auf Trockensubstanz.

2. A norganis cher P. $100 \mathrm{ccm}$. wässerigen Extractes $(=10 \mathrm{~g}$ feuchten Muskels $=5,84 \mathrm{~g}$ Trockensulbstanz) lieferten $0,092 \mathrm{~g}$ $\mathrm{Mg}_{2} \mathrm{P}_{2} \mathrm{O}_{7}$, entsprechend $0,2191 \% \mathrm{P}$, bezogen auf Trockensubstanz.

3. Organischer P (als Differenz berechnet) $=0,0488 \% \mathrm{P}$, bezogen auf Trockensubstanz.

4. Nucleone isenniederschlag. $5130 \mathrm{ccm}$. wässerigen Extractes $(=1010 \mathrm{~g}$ feuchten Muskels $=294 \mathrm{~g}$ Trockensubstanz) lieferten 7,807 $\mathrm{g}$ Eisenniederschlag mit 2,0\% $\mathrm{N}$ und $1,36 \% \mathrm{P}$.

Nucleon- $\mathrm{N}$ der Trockensubstanz $0,0544 \%$.

Nucleon-P » $\quad 0,0372 \%$. 
Versuch III. Junger, $161 / 2 \mathrm{~kg}$ schwerer Schäferhund, durch Verblutung. aus den Ciarotiden getödtet. Vorher mehrere Tage lang ruhig und während 18 Stunden nüchtern. Die Muskeln waren hellroth und sehr weich, so dass sie sehr schwer zu zerkleinern waren; der Fett'gehalt war mässig:

\section{Wassergehait.}

A. 16,8910 g feuchter Muskel lieferten $4,7036 \mathrm{~g}$ Trockensubstanz.

Wassergehalt des feuchten Muskels $=72,1 \%$.

B. $19,0990 \mathrm{~g}$ feuchter Muskel lieferten

0,2945 g Trockensubstanz.

Wassergehalt des feuchten Muskels $=72,2 \%$. Mittel $\mathbf{7 2 , 1 5} \%$.

Daher Trockensubstanz des feuchten Muskels $\mathbf{2 7 , 8 5} \%$.

II. Gesammit-P.

A. $4,7035 \mathrm{~g}$ getrockneter Muskel gaben $0,14 \dot{0} 0 \mathrm{~g} \mathrm{Mg}_{2} \mathrm{P}_{2} \mathrm{O}_{7}=0,430 \% \mathrm{P}$.

B. $\tilde{5}, 294 \check{g} \mathrm{~g}$ getrockneter Muskel gaben 0,1 ว $60 \mathrm{~g} \mathrm{Mg}_{2} \mathrm{P}_{2} \mathrm{O}_{7}=0,411 \% \mathrm{P}$.

$$
\text { Mittel } \mathbf{0 , 4 2 0} \% \text {. }
$$

\section{Wässeriger Extract.}

A. $78 \check{g} \mathrm{~g}$ feuchten Muskels gaben $364 \check{\mathrm{ccm}}$. wässerigen Extractes.

1. G e s a m m t-P. $46,5 \mathrm{ccm}$. des wässerigen Extractes $(=10 \mathrm{~g}$ feuchten Muskels $=2,785 \mathrm{~g}$ trockenen Muskels) lieferten $0,580 \mathrm{~g} \quad \mathrm{Mg}_{2} \mathrm{P}_{\mathrm{z}} \mathrm{O}_{7}$, entsprechend $0,2908 \%$, bezogen auf Trockensubstanz.

2. Anorganischer P. $93 \mathrm{ccm}$. des wässerigen Extractes (=20 g feuchten Muskels $=5,570 \mathrm{~g}$ trockenen Muskels) lieferten 0,095 g $\mathrm{Mg}_{2} \mathrm{P}_{2} \mathrm{O}_{7}$, entsprechend 0,2380\% auf Trockensubstanz.

3. Organischer P (als Differenz berechnet) $=0,0528 \% \mathrm{P}$, bezogen auf Trockensubstanz.

4. $\mathrm{Nucle}$ on fehlt.

B. 1. Ges a m m t-P. $46, \tilde{\mathrm{c}} \mathrm{cm}$. des wässerigen Extractes (= $10 \mathrm{~g}$ feuchten Muskels $=2,785 \mathrm{~g}$ trockenen Muskels) lieferten $0,061 \check{\mathrm{g}} \mathrm{Mg}_{2} \mathrm{P}_{2} \mathrm{O}_{7}$, entsprechend $0,3080 \% \mathrm{P}$, bezogen auf Trockensubstanz.

2. Anorganischer P. $93 \mathrm{ccm}$. des wässerigen Extractes

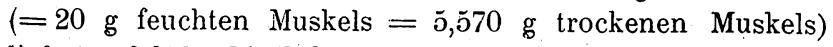
lieferten $0,093 \mathrm{~g} \mathrm{Mg}_{2} \mathrm{P}_{2} \mathrm{O}_{7}$, entsprechend $0,2333 \% \mathrm{P}$, bezogen auf Trockensubstanz.

3. Organis cher $\mathrm{P}($ Differenz) $=0,0747 \% \mathrm{P}$. 
4. Nucleoneis enniederschlag. $3366 \mathrm{ccm}$. wässerigen Extractes ( $=705 \mathrm{~g}$ feuchten Muskels $=196,8 \mathrm{~g}$ Trockensubstarı) lieferten $3,366 \check{\mathrm{g}}$ Eisenniederschlag mit $3,2 \% \mathrm{~N}$ und $1,65 \% \mathrm{P}$.

Nucleon-N der Trockensubstanz $0,051 \%$.

$$
\text { Nucleon-P > } \quad 0,0288 \% \text {. }
$$

Versuch IV. $40 \mathrm{~kg}$ schwerer Bernhardiner (Abart) von mittlerem Alter; durch Verblutung aus - den Carotiden getödtet. Vorher nicht besonders ruhend, aber während 12 Stunden nüchtern. Der Fettgehalt der Muskeln war gering.

\section{Wassergehalt.}

A. 6,2359 $\mathrm{g}$ feuchten Muskels lieferten

1,5155 g Trockensubstanz.

Wassergehalt des feuchten Muskels 75,7\%.

B. $5,1715 \mathrm{~g}$ feuchten Muskels lieferten

$1,2670 \mathrm{~g}$ Trockensubstanz.

Wassergehalt des feuchten Muskels 75,5\%.

\section{Mittel 75,6.}

Daher Trockensubstanz des feuchten Muskels $\mathbf{2 4 , 4} \%$.

\section{Gesammt-P.}

A. $1,5155 \mathrm{~g}$ getrockneter Muskel gaben

0,0415 g $\mathrm{Mg}_{2} \mathrm{P}_{2} \mathrm{O}_{7}=0,382 \% \mathrm{P}$.

B. 1,2670 g getrockneter Muskel gaben

$0,0415 \mathrm{~g}_{\mathrm{Mg}} \mathrm{P}_{2} \mathrm{O}_{7}=0,457 \% \mathrm{P}$.

$$
\text { Mittel } \mathbf{0 , 4 1 9} \% \text {. }
$$

\section{Wüsseriger Extract.}

A. $800 \mathrm{~g}$ feuchten Muskels gaben $3453 \mathrm{ccm}$. wässerigen Extractes.

1. Ges ammt-P. $43,2 \mathrm{ccm}$. des wässerigen Extractes $(=10 \mathrm{~g}$ feuchten Muskels $=2,44 \mathrm{~g}$ getrockneten Muskels) lieferten $0,0540 \mathrm{~g} \mathrm{Mg}_{2} \mathrm{P}_{2} \mathrm{O}_{7}$, entsprechend $0,307 \%$, P bezogen auf Trockensubstanz.

2. Anorganischer P. $86,4 \mathrm{ccm}$. des wässerigen Extractes (=20 g feuchten Muskels $=4,88 \mathrm{~g}$ trockenen Muskels) lieferten $0,07 \tilde{\mathrm{j}} \mathrm{g} \mathrm{Mg}_{2} \mathrm{P}_{2} \mathrm{O}_{7}$ entsprechend $0,215 \% \mathrm{P}$ bezogen auf Trockensubstanz.

3. Organischer $\mathrm{P}$ (Differenz) $=0,092 \% \quad \mathrm{P}$, bezogen auf Trockensubstanz.

4. Nucle oneisennieder s chlag. $3280,3 \mathrm{ccm}$. des wässerigen Extractes ( $=760 \mathrm{~g}$ feuchten Muskels $=188,6 \mathrm{~g}$ Trockensubstanz) lieferten $4,0963 \mathrm{~g}$ Eisenniederschlag mit $2,66 \% \mathrm{~N}$. und $1,76 \% \mathrm{P}$.

Nucleon-N der Trockensubstanz 0,057 \% .

Nucleon-P \ $\quad 0,0384 \%$.

Hoppe-Seyler's Zeitschrift f. physiol. Chemie. XXVIII. 
B. $800 \mathrm{~g}$ feuchten Muskels gaben $3905 \mathrm{ccm}$. wässerigen Extractes.

1. G e s a m m t-P. $48,8 \mathrm{ccm}$. des wässerigen Extractes $=10 \mathrm{~g}$ feuchten Muskels $=2,44 \mathrm{~g}$ trockenen Muskels) lieferten $0,058 \mathrm{~g} \mathrm{Mg}_{2} \mathrm{P}_{2} \mathrm{O}_{7}$, entsprechend $0,311 \% \mathrm{P}$, bezogen auf Trockensubstanz.

2. Anorganischer P. $97,6 \mathrm{ccm}$. des wässerigen Extractes ( $=20 \mathrm{~g}$ feuchten Muskels $=4,88 \mathrm{~g}$ trockenen Muskels) lieferten $0,076 \mathrm{~g} \mathrm{Mg}_{2} \mathrm{P}_{2} \mathrm{O}_{7}$, entsprechend $0,218 \% \mathrm{P}$, bezogen auf Trockensubstanz.

3. Organischer $\mathrm{P}$ (Differenz) $=0,137 \% \mathrm{P}$, bezogen auf Trockensubstanz.

4. $\mathrm{Nucl}$ eoneisennieder schlag. $3709,8 \mathrm{ccm}$. des wässerigen Extractes $(=760 \mathrm{~g}$ feuchten Muskels $=188 \mathrm{~g}$ Trockensubstanz) lieferten $3,5640 \mathrm{~g}$ Eisenniederschlag mit 2,8\% $\mathrm{N}$ und $2.02 \% \mathrm{P}$.

Nucleon-N der Trockensubstanz $0,529 \%$.

Nucleon-P » $\gg \quad 0,0382 \%$.

Versuch V. Alter, $35 \mathrm{~kg}$ schwerer Ziehhund; durch Verblutung aus den Carotiden getödtet. Vorher mehrere Tage lang ruhig und während der letzten 12 Stunden nüchtern. Die Muskeln waren dunkelroth und der Fettgehalt war mässig.

\section{Wassergehalt.}

A. $11,3115 \mathrm{~g}$ feuchter Muskel lieferten $2,8530 \mathrm{~g}$ Trockensubstanz.

Wassergehalt des feuchten Muskels $=74,7 \%$.

B. 13,2705 g feuchter Muskel lieferten

$3,0815 \mathrm{~g}$. Trockensubstanz.

Wassergehalt des feuchten Muskels 75,9\%

$$
\text { Mittel }=\mathbf{7 5 , 3} \% \text {. }
$$

Daher Trockensubstanz des feuchten Muskels $=\mathbf{2 4 , 7} \%$.

\section{Gesammt-P.}

A. $2,8530 \mathrm{~g}$ getrockneter Muskel gaben $0,0778 \mathrm{~g} \mathrm{Mg}_{8} \mathrm{P}_{8} \mathrm{O}_{i}=0,378 \% \mathrm{P}$.

B. $3,081 \tilde{\mathrm{g}} \mathrm{g}$ getrockneter Muskel gaben 0,0778 g $^{M_{2}} \mathrm{P}_{2} \mathrm{O}_{7}=0,350 \% \mathrm{P}$.

$$
\text { Mittel }=\mathbf{0 , 3 6 7} \% \text {. }
$$

\section{Wässeriger Extract.}

A. $800 \mathrm{~g}$ feuchten Muskels gaben $4162 \mathrm{ccm}$. wässerigen Extractes.

1. Ges a mmt-P. $52 \mathrm{ccm}$. des wässerigen Extractes $(=10 \mathrm{~g}$ feuchten Muskels $=2,47 \mathrm{~g}$ trockenen Muskels) lieferten $0,0 \check{10} \mathrm{~g} \mathrm{Mg}_{2} \mathrm{P}_{2} \mathrm{O}_{i}$, entsprechend $0,287 \% \mathrm{P}$, bezogen auf Trockensubstanz. 
2. Anorganischer P. $104 \mathrm{ccm}$. des wässerigen Extractes ( $=20 \mathrm{~g}$ feuchten Muskels $=4.54 \mathrm{~g}$ trockenen Muskels $)$ lieferten 0,0705 g $\mathrm{Mg}_{2} \mathrm{P}_{2} \mathrm{O}_{\tau}$, entsprechend $0,198 \% \mathrm{P}$, bezogen auf Trockensubstanz.

3. Organischer P (als Differenz berechnet) $=0,089 \% \mathrm{P}$, bezogen auf Trockensubstanz.

4. Nucleoneisennieder schlag. 3944 ccm. des wässerigen Extractes $(=760 \mathrm{~g}$ feuchten Muskels $=187,7 \mathrm{~g} \mathrm{P}$ Trockensubstanz) lieferten $5,1855 \mathrm{~g}$ Eisenniederschlag mit $2,72 \% \mathrm{~N}$ und $1,33 \% \mathrm{P}$.

Nucleon- $\mathrm{N}$ der Trockensubstanz $=0,074 \%$.

Nucleon-P » $\quad=0,042 \%$.

B. $800 \mathrm{~g}$ feuchten Muskels gaben $4461 \mathrm{ccm}$. wässerigen Extractes.

1. Ge s a m m t-P. $55,7 \mathrm{ccm}$. des wässerigen Extractes (=10 g feuchten Muskels $=2,47 \mathrm{~g}$ trockenen Muskels) lieferten $0,0545 \mathrm{~g} \mathrm{Mg}_{2} \mathrm{P}_{2} \mathrm{O}_{7}$, entsprechend $0,307 \% \mathrm{P}$, bezogen auf Trockensubstanz.

2. Anorganis cher P. $111,4 \mathrm{ccm}$. des wässerigen Extractes $(=20 \mathrm{~g}$ feuchten Muskels $=4,54 \mathrm{~g}$ trockenen Muskels) lieferten $0,079 \mathrm{~g} \mathrm{Mg}_{2} \mathrm{P}_{2} \mathrm{O}_{7}$, entsprechend $0,222 \% \mathrm{P}$, bezogen auf Trockensubstanz.

3. Organischer P (als Differenz berechnet) $=0,085 \% \mathrm{P}$, bezogen auf Trockensubstanz.

4. Nucle on eisen nieder s chlag. $4238,8 \mathrm{ccm}$. des wässerigen Extractes $(=760 \mathrm{~g}$ feuchten Muskels $=187,7 \mathrm{~g}$ Trockensubstanz) lieferten 6,1480 g Eisenniederschlag mit $2,20 \% \mathrm{~N}$ und $1,39 \% \mathrm{P}$.

Nucleon-N der Trockensubstanz $=0,071 \%$.

Nucleon-P $>\quad=0,045 \%$.

Versuch VI. Alter, $40 \mathrm{~kg}$. schwerer Ziehhund, arbeitete 20 Min. lang in der Tretmühle, dann musste das Experiment unterbrochen werden, da hochgradige Dyspnoe eintrat; darauf wurde das Thier durch Verblutung aus den Carotiden getödtet; das Blut war sehr dunkel; die sofort nachher herauspräparirten Muskeln zeigten keine Todtenstarre; ihr Fettgehalt war mässig.

\section{Wassergehalt.}

A. $8,7185 \mathrm{~g}$ feuchter Muskel lieferten 2,3730 g Trockensubstanz.

Wassergehalt des feuchten Muskels $=72,7 \%$.

B. $13,8925 \mathrm{~g}$ feuchter Muskel lieferten

3,8213 g Trockensubstanz.

Wassergehalt des feuchten Muskels $=72,4 \%$.

Mittel $\mathbf{7 2 , 5 5} \%$.

Daher Trockensubstanz des feuchten Muskels $=\mathbf{2 7 , 4 5} \%$. 


\section{Gesammt-Phosphor.}

A. 2,3730 g getrockneter Muskel gaben $0,0570 \mathrm{~g} \mathrm{Mg}_{2} \mathrm{P}_{2} \mathrm{O}_{7}=0,335 \% \mathrm{P}$.

B. $3,8213 \mathrm{~g}$ getrockneter Muskel gaben $0,0900 \mathrm{~g} \mathrm{Mg}_{2} \mathrm{P}_{2} \mathrm{O}_{7}=0,329{ }_{10} \mathrm{P}$.

$$
\text { Mittel }=\mathbf{0 , 3 3 2} \% \text {. }
$$

\section{Wässeriger Extract.}

A. $800 \mathrm{~g}$ feuchten Muskels gaben $3800 \mathrm{ccm}$. wässerigen Extractes.

1. Ge s a m m t-P. $47,5 . \mathrm{ccm}$. des wässerigen Extractes $(=10 \mathrm{~g}$ feuchten Muskels $=2,74 \check{g} \mathrm{~g}$ trockenen Muskels) lieferten $0,0475 \mathrm{~g} \mathrm{Mg}_{2} \mathrm{P}_{2} \mathrm{O}_{7}$, entsprechend $0,240 \% \mathrm{P}$, bezogen auf Trockensubstanz.

2. Anorganischer P. $95 \mathrm{ccm}$. des wässerigen Extractes (=20 g feuchten Muskels $=5,490 \mathrm{~g}$ trockenen Muskels) lieferten $0,0723 \mathrm{~g} \mathrm{Mg}_{2} \mathrm{P}_{2} \mathrm{O}_{7}$, entsprechend $0,18 \check{0} \% \mathrm{P}$, bezogen auf Trockensubstanz.

3. Organischer P (als Differenz berechnet) $=0,057 \% \mathrm{P}$, bezogen auf Trockensubstanz.

4. Nucleoneisenniederschlag. $3610 \mathrm{ccm}$. wässerigen Extractes ( $=760 \mathrm{~g}$ feuchten Muskels $=208,6 \mathrm{~g}$ Trockensubstanz) lieferten $3,3315 \mathrm{~g}$ Eisenniederschlag mit $3,0 \% \mathrm{~N}$ und $1,88 \% \mathrm{P}$.

Nucleon-N der Trockensubstanz $=0,0478 \%$.

Nucleon.P » $>\quad=0,0300 \%$.

B. $800 \mathrm{~g}$ feuchten Muskels gaben $3676 \mathrm{ccm}$. wässerigen Extractes.

1. Ge sam t-P. $46 \mathrm{ccm}$. des wässerigen Extractes $(=10 \mathrm{~g}$ feuchten Muskels $=2,74 \hat{\mathrm{O}} \mathrm{g}$ trockenen Muskels) lieferten $0,0505 \mathrm{~g} \mathrm{Mg}_{2} \mathrm{P}_{2} \mathrm{O}_{7}$, entsprechend $0,255 \% \mathrm{P}$, bezogen auf Trockensubstanz.

2. Anorganischer P. $92 \mathrm{ccm}$. des wässerigen Extractes (= 20. $\mathrm{g}$ feuchten Muskels $=5,490 \mathrm{~g}$ trockenen Muskels) lieferten $0,07: 30 \mathrm{~g} \mathrm{Mg}_{2} \mathrm{P}_{2} \mathrm{O}_{7}$, entsprechend $0,185 \% \mathrm{P}$, bezogen auf Trockensubstanz.

3. Organis cher P (als Differenz berechnet) $=0,069 \% \mathrm{P}$, bezogen auf Trockensubstanz.

4. Nucleoneisennieder schlag. $3472 \mathrm{ccm}$. des wässerigen Extractes ( $=760 \mathrm{~g}$ feuchten Muskels $=208,6 \mathrm{~g}$ Trockensubstanz) lieferten $2,4895 \mathrm{~g}$ Eisenniederschlag mit $3,45 \% \mathrm{~N}$ und $1,87 \% \mathrm{P}$.

Nucleon-N der Trockensubstan $z=0,0415 \%$.

Nucleon-P $\gg \quad=0,0298 \%$. 


\section{Versuche an ermüdeten Hunden.}

Versuch I. $18 \mathrm{~kg}$ schwerer Schäferhund von mittlerem Alter, 4x Stunden nüchtern; arbeitete $3_{1 / 2}^{11}$ Stunden; darauf verblutet aus den Carotiden. Das Blut war nicht so dunkel wie in Vers. VI (S. 545). Die Todtenstarre trat circa 20 Minuten nach dem Tode ein. Muskeln dunkelroth; Fettgehalt mässig.

\section{Wassergehalt.}

A. 10,2805 g feuchter Muskel lieferten

2,8230 g Trockensubstanz.

Wassergehalt des feuchten Muskels $=72,54 \%$.

B. $12,4530 \mathrm{~g}$ feuchten Muskel lieferten

3,3835 g Trockensubstanz.

Wassergehalt des feuchten Muskels $72,83^{\circ}{ }_{1}$.

Mittel $\mathbf{7 2}, 68 \%$.

Daher Trockensubstanz des feuchten Muskels $=27,32 \%$.

\section{Gesammt-P.}

A. $2,8230 \mathrm{~g}$ getrockneter Muskel gaben $0,880 \quad \mathrm{~g} \mathrm{Mg}_{2} \mathrm{P}_{2} \mathrm{O}_{7}=0,436 \% \mathrm{P}$.

B. $3,3835 \mathrm{~g}$ getrockneter Muskel gaben $1,040 \mathrm{~g} \mathrm{Mg}_{2} \mathrm{P}_{2} \mathrm{O}_{7}=0,428 \mathrm{P}$.

\section{Mittel $\mathbf{0 , 4 3 2} \%$.}

\section{Wässeriger Extract.}

A. $700 \mathrm{~g}$ feuchten Muskels gaben $3: 385 \mathrm{ccm}$. des wässerigen Extractes.

1. Gesammt-P. $48 \mathrm{ccm}$. des wässerigen Extractes $(=10 \mathrm{~g}$ feuchten Muskels $=2,732 \mathrm{~g}$ Trockensubstanz) lieferten $0,0555 \mathrm{~g} \mathrm{Mg}_{2} \mathrm{P}_{2} \mathrm{O}_{\pi}$, entsprechend $0,281 \% \mathrm{P}$, bezogen auf Trockensubstanz.

2. Anorganischer P. $96 \mathrm{ccm}$. des wässerigen Extractes (= $20 \mathrm{~g}$ feuchten Muskels $=5,464 \mathrm{~g}$ Trockensubstanz) lieferten $0,0930 \mathrm{~g} \mathrm{Mg}_{2} \mathrm{P}_{2} \mathrm{O}_{7}$, entsprechend $0,237 \% \mathrm{P}$, bezogen auf Trockensubstanz.

3. Organischer P (als Differenz berechnet) $=0,044 \% \mathrm{P}$, bezogen auf Trockensubstanz.

4. $\mathrm{Nucleon}$ eisenniederschlag $3193 \mathrm{ccm}$. des wässerigen Extractes $(=660 \mathrm{~g}$ feuchten Muskels $=180 \mathrm{~g}$ Trockensubstanz) lieferten $3,3740 \mathrm{~g}$ Eisenniederschlag mit $2,2 \% \mathrm{~N}$ und $1,68 \% \mathrm{P}$.

Nucleon-N der Trockensubstanz $=0,043 \%$.

Nucleon-P » $\quad=0,033 \%$.

B. $700 \mathrm{~g}$ feuchten Muskels gaben $3605 \mathrm{ccm}$. wässerigen Extractes.

1. Gesammt-P. $50,1 \mathrm{ccm}$. des wässerigen Extractes $(=10 \mathrm{~g}$ feuchten Muskels $=2,732 \mathrm{~g}$ Trockensubstanz) lieferten 
$0,0510 \mathrm{~g} \quad \mathrm{Mg}_{2} \mathrm{P}_{2} \mathrm{O}_{7}$ entsprechend $0,259 \% \mathrm{P}$ bezogen auf Trockensubstanz.

2. Anorganischer P. $100,2 \mathrm{ccm}$. des wässerigen Extractes $\left(=5,464 \mathrm{~g}\right.$ Trockensubstanz) lieferten $0,0855 \mathrm{~g} \mathrm{Mg}_{2} \mathrm{P}_{2} \mathrm{O}_{7}$ entsprechend, 0,229\% $\mathrm{P}$ bezogen auf Trockensubstanz.

3. Organischer $\mathrm{P}$ (als Differenz berechnet) $=0,030 \% \mathrm{P}$, bezogen auf Trockensubstanz.

4. Nucleoneisenniederschlag $3402,6 \mathrm{ccm}$. des wässerigen Extractes $(=660 \mathrm{~g}$ feuchten Muskels $=180 \mathrm{~g}$ Trockensubstanz) lieferten $3,9690 \mathrm{~g}$ Eisenniederschlag mit $2,1 \% \mathrm{~N}$ und $1,66 \% \mathrm{P}$.

Nucleon-N der Trockensubstanz $=0,046 \%$.

Nucleon-P $>\quad=0,036 \%$.

Versuch II. Junge, $14^{1 / 2} \mathrm{~kg}$ schwere Schäferhündin; 12 Stunden lang nüchtern. Arbeitete in der Tretmühle $44^{1 / 4}$ Stunden; verblutet; die Todtenstarre kam 15 Minuten nach dem Tode zum Vorschein. Blut dunkel. Der Fettgehalt war mässig.

\section{Wassergehalt.}

A. Verloren.

B. $16,3050 \mathrm{~g}$ feuchter Muskel lieferten

4,1685 g Trockensubstanz.

Wassergehalt des feuchten Muskels $74,4 \%$.

Daher Trockensubstanz des feuchten Muskels $=25,6 \%$.

\section{Gesammt-P.}

A. Verloren.

B. $4,1685 \mathrm{~g}$ getrockneter Muskel gaben

$0,1440 \mathrm{~g} \mathrm{Mg}_{2} \mathrm{P}_{2} \mathrm{O}_{7}=0,4833 \% \mathrm{P}$.

$$
\text { Mittel } \mathbf{0 , 4 8 3} \% \text {. }
$$

\section{Wusseriger Extract.}

A. $600 \mathrm{~g}$. feuchten Muskels gaben $2935 \mathrm{ccm}$. wässerigen Extractes.

1. Ge sammt-P. $49 \mathrm{ccm}$. des wässerigen Extractes $(=10 \mathrm{~g}$ feuchten Muskels $=2,56 \mathrm{~g}$ Trockensubstanz) lieferten 0,0545 g $\mathrm{Mg}_{2} \mathrm{P}_{z} \mathrm{O}_{7}$, entsprechend $0,296 \% \mathrm{P}$, bezogen auf Trockensubstanz.

2. A norganischer P. $98 \mathrm{ccm}$. des wässerigen Extractes $(=20 \mathrm{~g}$ feuchten Muskels = $5,12 \mathrm{~g}$ Trockensubstanz) lieferten 0,0920 g $\mathrm{Mg}_{2} \mathrm{P}_{2} \mathrm{O}_{7}$, entsprechend $0,250 \% \mathrm{P}$, bezogen auf Trockensubstanz.

3. Organischer $\mathrm{P}$ (Differenz) $=0,046 \% \mathrm{P}$, bezogen auf Trockensubstanz.

4. Nucleoneisenniederschlag $2739 \mathrm{ccm}$. des wässerigen Extractes $(=560 \mathrm{~g}$ feuchten Muskels $=143,3 \mathrm{~g}$ Trocken- 
substanz) lieferten $3,3845 \%$ Eisenniederschlag mit $3,15 \% \mathrm{~N}$ und $1,39 \% \mathrm{P}$.

Nucleon-N der Trockensubstanz $=0,074 \%$.

Nucleon-P » $>0,032 \%$.

B. $6100 \mathrm{~g}$ feuchten Muskels gaben $2864 \mathrm{ccm}$. des wässerigen Extractes.

1. Ges am m t-P. 47, $5 \mathrm{ccm}$. des Extractes (= $10 \mathrm{~g}$ feuchten Muskels $=2,56 \mathrm{~g}$ Trockensubstanz) lieferten $0,0 \check{3} 35 \mathrm{~g} \mathrm{Mg}_{2} \mathrm{P}_{2} \mathrm{O}_{7}$, entsprechend $0,289 \% \mathrm{P}$, bezogen auf Trockensubstanz.

2. Anorgan is cher P. $95 \mathrm{ccm}$. des Extractes $(=20 \mathrm{~g}$ feuchten Muskels $=\tilde{0}, 12 \mathrm{~g}$ Trockensubstanz) lieferten $0,091 \check{\mathrm{g}} \mathrm{Mg}_{\mathbf{2}} \mathrm{P}_{\mathbf{2}} \mathrm{O}_{\mathbf{7}}$ entsprechend $0,250 \% \mathrm{P}$, bezogen auf Trockensubstanz.

3. Organischer P (als Differenz berechnet) $=0,039 \% \mathrm{P}$, bezogen auf Trockensubstanz.

4. $\mathrm{Nucl}$ eoneis enniederschlag. $2674 \mathrm{ccm}$. des wässerigen Extractes ( $=560 \mathrm{~g}$ feuchten Muskels $=143,3 \mathrm{~g}$ Trockensubstanz) lieferten $2,6345 \mathrm{~g}$ Eisenniederschlag mit $2,85 \% \mathrm{~N}$ und $1,57 \% \mathrm{P}$.

Nucleon-N der Trockensubstanz $=0,052 \%$.

Nucleon-P » $\gg, 0,030 \%$.

Versuch III. Junge, $18 \frac{1}{2} \mathrm{~kg}$ schwere Schäferhündin; 12 Stunden lang nüchtern. Arbeitete in der Tretmühle 6 Stunden. Die Verblutung lauerte eine Stunde. Die Todtenstarre kam später ( 45 Minuten) wie in Nr. II zum Vorschein. Der Fettgehalt war mässig.

\section{Wassergehalt.}

A. $12,2290 \mathrm{~g}$ feuchten Muskels lieferten

$3,0400 \mathrm{~g}$ Trockensubstanz.

Wassergehalt des feuchten Muskels 75,74\%.

B. $12,0145 \mathrm{~g}$ feuchter Muskel lieferten 3,0850 g Trockensubstanz.

Wassergehalt des feuchten Muskels $=7 \check{0}, 74 \%$.

$$
\text { Mittel }=\mathbf{7 5 , 4 4}
$$

Daher Trockensubstanz des feuchten Muskels $=\mathbf{2 4 , 2 6} \%$.

\section{Gesammt-P.}

A. 3,0400 g getrockneten Muskels lieferten 0,1080 g. $\mathrm{M}_{\mathbf{8}} \mathrm{P}_{\mathbf{2}} \mathrm{O}_{\mathbf{7}}$ $=0,496 \% \mathrm{P}$.

B. $2,915 \mathrm{~g}$ getrockneten Muskels lieferten $0,0975 \mathrm{~g} \quad \mathrm{Mg}_{2} \mathrm{P}_{2} \mathrm{O}_{7}$ $=0,466 \% \mathrm{P}$.

$$
\text { Mittel } \mathbf{0 , 4 8 1} \% \mathrm{P} \text {. }
$$

\section{Wässeriger Extract.}

A. $600 \mathrm{~g}$ feuchten Muskels gaben $3300 \mathrm{ccm}$ : wässerigen Extractes.

1. Gesammt-P. ว̆ $\mathrm{ccm}$. des wässerigen Extractes $(=10 \mathrm{~g}$ feuchten Muskels $=2,456 \mathrm{~g}$ Trockensubstanz) lieferten 0,0510 g $\quad \mathrm{Mg}_{2} \mathrm{P}_{2} \mathrm{O}_{7}$, entsprechend $0,289 \% \mathrm{P}$, bezogen auf Trockensubstanz. 
2. Anorganischer P. $110 \mathrm{ccm}$. des wässerigen Extractes (=20 g feuchten Muskels $=4.912 \mathrm{~g}$ Trockensubstanz) lieferten $0,0950 \mathrm{~g} \mathrm{Mg}_{2} \mathrm{P}_{2} \mathrm{O}_{7}$ entsprechend $0,268 \% \mathrm{P}$, bezogen auf Trockensubstanz.

3. Organischer $\mathrm{P}$ (als Differenz berechnet) $=0,021 \% \mathrm{P}$, bezogen auf Trockensubstanz.

4. Nucleoneisenniederschlag $3080 \mathrm{ccm}$. des wässerigen Extractes $(=560 \mathrm{~g}$ feuchten Muskels $=137.5 \mathrm{~g}$ Trockensubstanz) lieferten $4,0650 \mathrm{~g}$ Eisenniederschlag mit $3,2 \% \mathrm{~N}$ und $0,59 \% \mathrm{P}$.

Nucleon-N der Trockensubstanz $=0,094 \%$.

Nucleon-P » $\gg=0,016 \%$.

B. $600 \mathrm{~g}$ feuchten Muskels gaben $2775 \mathrm{ccm}$. wässerigen Extractes.

1. Ges a mmt-P. $46,2 \mathrm{ccm}$. des wässerigen Extractes $(=10 \mathrm{~g}$ feuchten Muskels $=2,456 \mathrm{~g}$ Trockensubstanz) lieferten 0,0495 g $\mathrm{Mg}_{2} \mathrm{P}_{2} \mathrm{O}_{7}$, entsprechend $0,280 \% \mathrm{P}$, bezogen auf Trockensubstanz.

2. Anorganischer P. $92,4 \mathrm{ccm}$. des wässerigen Extractes (=20 $\mathrm{g}$ feuchten Muskels $=4,912 \mathrm{~g}$ Trockensubstanz) lieferten $0,0920 \mathrm{~g} \mathrm{Mg}_{2} \mathrm{P}_{2} \mathrm{O}_{-7}$, entsprechend $0,260 \% \mathrm{P}$, bezogen auf Trockensubstanz.

3. Organischer P (als Differenz berechnet) $=0,020 \% \mathrm{P}$, bezogen auf Trockensubstanz.

4. Nucleoneisenniederschlag. $2590,4 \mathrm{ccm}$. des wässerigen Extractes $(=560 \mathrm{~g}$ feuchten Muskels $=137,5 \mathrm{~g}$ Trockensubstanz) lieferten $4,1805 \mathrm{~g}$ Eisenniederschlag mit $3,38 \% \mathrm{~N}$ und $0,50 \% \mathrm{P}$.

Nucleon- $\mathrm{N}$ der Trockensubstanz $=0,102 \%$.

Nucleon-P 》 $>0,015 \%$.

Versuch 1V. $16^{1 / 2} \mathrm{~kg}$ schwerer Bullenbeisser von mittlerem Alter, 12 Stunden lang nüchtern; arbeitete 5\% Stunden; darauf verblutet aus den Carotiden. Die Todtenstarre trat circa 30 Minuten nach dem Tode ein. Fettgehalt gross.

\section{Wassergehalt.}

A. $11, \check{05} 59 \mathrm{~g}$ g feuchter Muskel lieferten $3,3605 \mathrm{~g}$ Trockensubstanz. Wassergehalt des feuchten Muskels $=70,9 \%$.

B. $12,8465 \mathrm{~g}$ feuchter Muskel lieferten 3,9655 g Trockensubstanz.

Wassergehalt des feuchten Muskels $=70,7 \%$.

\section{Mittel $\mathbf{7 0 , 8}$}

Daher Trockensubstanz des feuchten Muskels $=\mathbf{2 9 , 2} \%$.

\section{Gesammtphosphor.}

A. 3,3605 g getrockneter Muskel gaben $0,0930 \mathrm{~g} \mathrm{Mg}_{2} \mathrm{P}_{2} \mathrm{O}_{7}=0,384 \% \mathrm{P}$.

B. 3,9655 g getrockneter Muskel gaben $0,1145 \mathrm{~g} \mathrm{Mg}_{2} \mathrm{P}_{2} \mathrm{O}_{7}=0,376 \% \mathrm{P}$. Mittel $\mathbf{0 , 3 8 0} \% \mathrm{P}$. 


\section{Wässeriger Extract.}

A. $600 \mathrm{~g}$ feuchten Muskels gaben $3750 \mathrm{ccm}$. wässerigen Extractes.

1. Ges a m m t-P. $62,5 \mathrm{ccm}$. des wässerigen Extractes $(=10 \mathrm{~g}$ feuchten Muskels $=2,92 \mathrm{~g}$. Trockensubstanz) lieferten $0,0535 \mathrm{~g} \mathrm{Mg}_{2} \mathrm{P}_{2} \mathrm{O}_{7}$, entsprechend $0,252 \% \mathrm{P}$, bezogen auf Trockensubstanz.

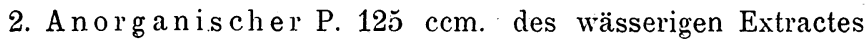
(=20 g feuchten Muskels $=5,84 \mathrm{~g}$ Trockensubstanz) lieferten $0,0905 \mathrm{~g} \mathrm{Mg}_{2} \mathrm{P}_{2} \mathrm{O}_{\overline{7}}$, entsprechend $0,215 \% \mathrm{P}$, bezogen auf Trockensubstanz.

3. Organischer P (als Differenz berechnet) $=0,037 \% \mathrm{P}$, bezogen auf Trockensubstanz.

4. Nucleon e is en nieders chlag. $3562 \mathrm{ccm}$. des wässerigen Extractes $(=570 \mathrm{~g}$ feuchten Muskels $=166,4 \mathrm{~g}$ Trockensubstanz) lieferten $4,7060 \mathrm{~g}$ Eisenniederschlag mit 2,45 \% N und $0,73 \% \mathrm{P}$.

Nucleon-N der Trockensubstanz 0,069\%.

Nucleon-P > $\quad 0,020 \%$.

B. $450 \mathrm{~g}$ feuchten Muskels gaben $2925 \mathrm{ccm}$. des Extractes.

1. Gesammt-P. $65 \mathrm{ccm}$. des wässerigen Extractes $(=10 \mathrm{~g}$ feuchten Muskels $=2,92 \mathrm{~g}$ Trockensubstanz) lieferten $0,055 \mathrm{~g}$ $\mathrm{Mg}_{2} \mathrm{P}_{2} \mathrm{O}_{7}$, entsprechend $0,260 \% \mathrm{P}$, bezogen auf Trockensubstanz.

2. Anorganischer P. $130 \mathrm{ccm}$. des wässerigen Extractes (=20 g feuchten Muskels $=5,84 \mathrm{~g}$ Trockensubstanz) lieferten $0,0965 \mathrm{~g} \mathrm{Mg}_{2} \mathrm{P}_{2} \mathrm{O}_{7}$, entsprechend $0,231 \% \mathrm{P}$, bezogen auf Trockensubstanz.

3. Organischer $\mathrm{P}$ (Differenz) $=0,029 \% \mathrm{P}$, bezogen auf Trockensubstanz.

4. Nucleoneisenniederschlag $2730 \mathrm{ccm}$. des wässerigen Extractes ( $=420 \mathrm{~g}$ feuchten Muskels $=122,6 \mathrm{~g}$ Trockensubstanz) lieferten $3,1530 \mathrm{~g}$ Eisenniederschlag mit $2,25 \% \mathrm{~N}$ und $0,60 \% \mathrm{P}$.

Nucleon-N der Trockensubstanz 0,057\%.

Nucleon-P • $\quad 0,0155 \%$.

Der besseren Uebersichtlichkeit halber sind die Mittelwerthe aus den sämmtlichen vorerwähnten P-Bestimmungen in den nachfolgenden Tabellen zusammengestellt. Ich gebe auch die Zahlen für die Verhältnisse, in welchen die wichtigsten dieser Werthe zu einander stehen. 


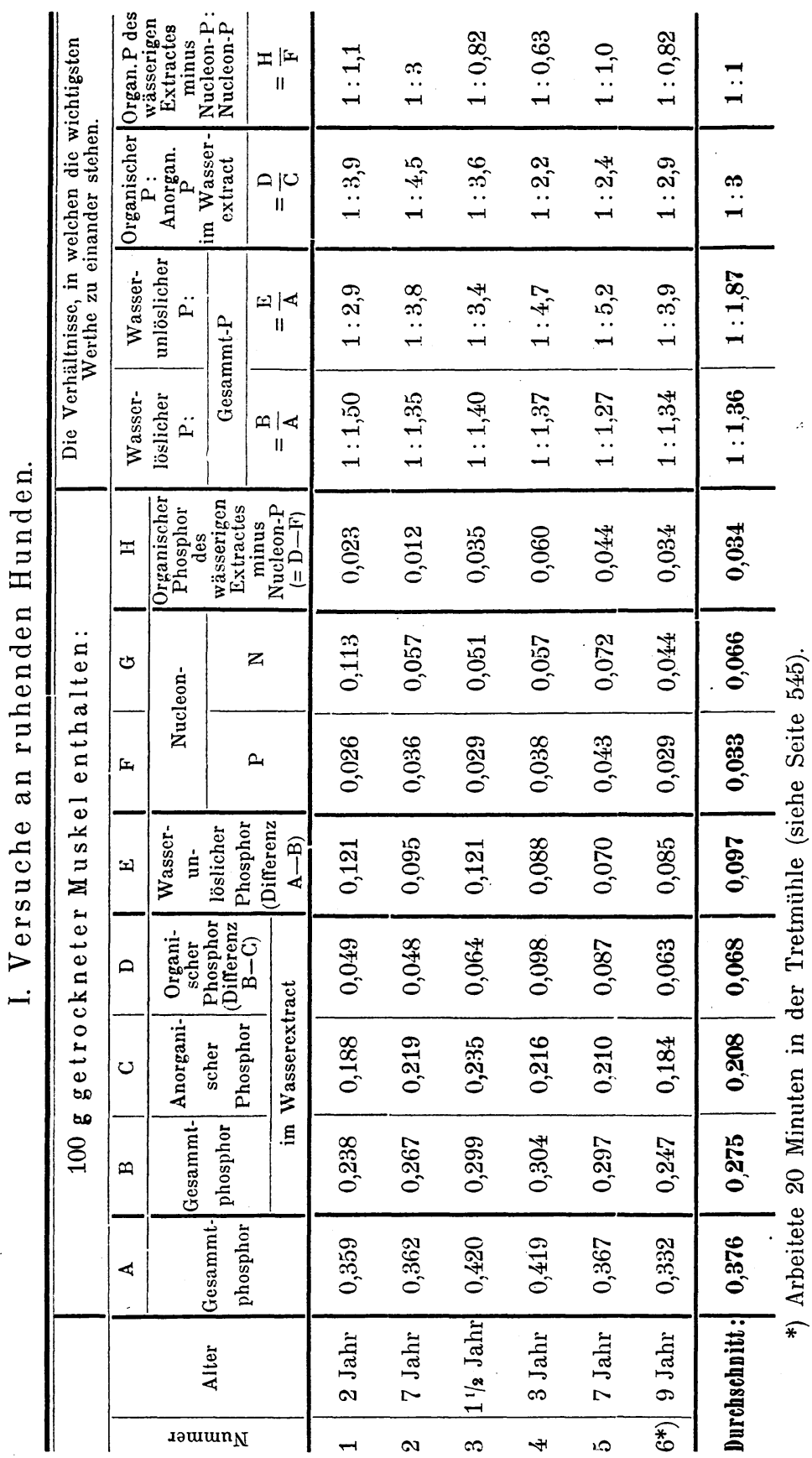




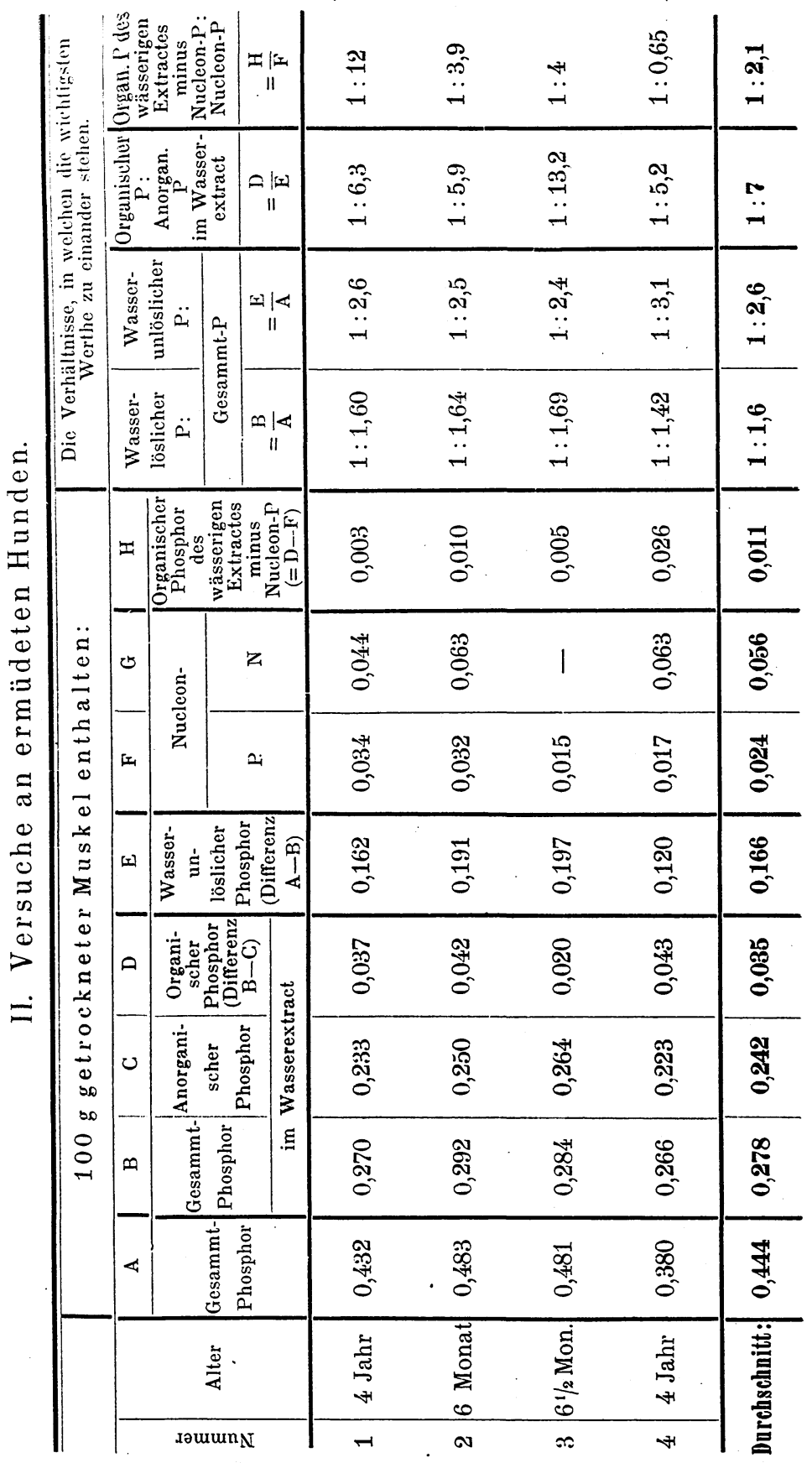


Ein Blick auf diese Tabelle belehrt uns zunächst, dass zwar der Gesammtphosphorgehalt im ruhenden Hundemuskel beträchtlich variirt, dass aber die Verhältnisse der verschiedenen Gruppen unter einander ziemlich constant bleiben.

Der Gesammtphosphor (A Tab. I) hat einen Durchschnittswerth von $0,376 \% \mathrm{P}$ und variirt zwischen $0,332 \% \mathrm{P}$ und $0,420 \% \mathrm{P}$, wobei in der Regel die grösseren Zahlen von jungen, die niedrigeren von alten Hunden stammen. Nur der Hund in Versuch I zeigt niedrige Werthe, obgleich er ein junges Thier war.

Der Phosphor, welcher in den wässerigen Extract übergeht (B Tab. I), bildet im Allgemeinen $73 \%$ des Gesammtphosphors, und von dem Phosphor des wässerigen Extractes selbst sind wieder $75 \%$ anorganisch und $25 \%$ organisch. Von diesem letzteren organisch gebundenen Phosphor, welcher in den wässerigen Extract übergeht, sind durchschnittlich $50 \%$ Nucle onphosphor (wie aus der letzten Columne der Tabelle I ersichtlich ist). Die übrigen $50 \%$ gehören wahrscheinlich nur zum kleinen Theile der Inosinsäure an, welche zwar, wie Liebig ${ }^{1}$ ) und Haiser ${ }^{2}$ ) gezeigt haben, in Wasser löslich ist, und welche nach Haiser organisch gebundenen Phosphor besitzt, von der aber der Muskel nur äusserst geringe Mengen enthält.

Was für Stoffe sind es nun neben der Inosinsäure, welche diesen nicht durch Eisen fällbaren organischen Phosphor enthalten? Zunächst war daran zu denken, dass vielleicht die Eisenfällung des Nucleons unvollständig gewesen wäre. Zur Prüfung dieser Möglichkeit wurden zwei Versuche in der nachstehenden Weise angestellt. Nachdem man den Nucleoneisenniederschlag sich hatte setzen lassen, wurde die darüber befindliche Flüssigkeit abgegossen und in einer grossen Schale zum Kochen erhitzt. Dazu wurde dann verdünntes Ammoniak gegeben, bis die Reaction eben alkalisch wurde, und $\mathrm{FeCl}_{3}$ Lösung in geringem Ueberschuss hinzugefügt. Es bildete sich

1) Annalen der Chemie und Pharmacie, Bd. LXII, S. 317.

2) Monatshefte für Chemie. 1895́. S. 190. 
ein nenerlicher Niederschlag. Derselbe lieferte nach dem Verschmelzen mit Aetznatron und Salpeter keine Spur Phosphorsäure und enthielt Stickstoff, der jedenfalls von Albumosen herrührt.

Daraus ergibt sich, dass die Fällung des Nucleons eine vollständige war. Der restliche organische Phosphor des wässerigen Extractes ist daher nicht auf Rechnung des nichtgefällten Nucleons zu-setzen., Ebenso wenig dürfte er wohl aus dem Nuclein oder Lecithin des Muskels stammen. Es erscheint demnach wahrscheinlich, dass der Hundemuskel ausser dem Nucleon noch andere wasserlösliche Verbindungen enthält, in welchen organisch gebundener Phosphor vorhanden ist.

Der Nucleonphosphor, ausgedrückt in Procenten des getrockneten Muskels, ist unserer Tabelle zufolge eine ziemlich constante Grösse; hingegen schwankt der Nucleonstickstoff etwas mehr.

Gehen wir nun zu den Versuchen an ermüdeten Hunden über, so sehen wir, dass die hauptsächlichste, durch die Muskelarbeit bewirkte Veränderung im Phosphorgehalt des Muskels darin besteht, dass der organische Phosphor im wässerigen Extractsehr beträchtlich vermindert wird.

Diese Verminderung betrifft, wie die Tabelle lehrt, zum Theile den Phosphor des Nucleons, in der Hauptsache aber die anderen oben erwähnten organisch phosphorhaltigen Extractivstoffe des Muskels.

Besonders in den Versuchen III und IV ist der Phosphor des Nucleons sehr deutlich herabgesetzt (Tab. II F), also in jenen Versuchen, in welchen die Ermüdung am weitesten getrieben worden war. Diese Resultate stimmen mit denen, welche Siegfried ${ }^{1}$ ) bei arbeitenden Hunden erhalten, insofern überein, als in dem Versuch III von Siegfried, bei dem die Arbeit in der Leistung eines 1,5-stündigen Weges bestand, nur wenig Nucleon verbraucht wurde, während die tetanisirten Muskeln beträchtlichen Nucleonschwund aufwiesen.

1) Diese Zeitschrift, Bd. XXI, S. 377. 
Entsprechend der Verminderung des gesammten wasserlöslichen organischen Phosphors zeigt sich eine beträchtliche Zunahme in den wasserlöslichen anorganischen Phosphaten (Natrium- und Kaliumphosphat), und es ist sehr wahrscheinlich, dass in eben dieser Form der verbrauchte Phosphor auch den Muskel verlässt und in das Blut übergeht, um durch die Nieren ausgeschieden zu werden. Ich beabsichtige, auf verschiedene and̦ere Weise diese Sache noch weiter $\mathrm{zu}$ verfolgen.

Der Gesammtphosphor des wässerigen Extractes bleibt ziemlich derselbe wie bei den ruhenden Hunden, nur das Verhältniss zwischen dem organischen und anorganischen Phosphor desselben $\left(\frac{\mathrm{D}}{\mathrm{C}}\right.$ Tab. II) ist anstatt $1: 3$ (bei ruhenden Hunden) $1: 5$ und $1: 6$ in den Versuchen I, II und IV, und sogar $1: 13$ in Versuch III.

Sehr auffallend ist, dass der. Gesammtphosphor des Muskels durch die Arbeit zuzunehmen scheint (siehe A Tab. II); besonders deutlich ist dies in Experiment II und III, wo indessen die Zunahme, theilweise wenigstens, mit der Thatsache zusammenhängen kann, dass die Thiere sehr jung (5-6 Monate) waren und also - wie Bunge gezeigt hat relativ mehr anorganische Substanzen (also auch Erdphosphate) in ihren Geweben enthalten müssen, als die älteren Thiere.

Da nun bei der Thätigkeit der Muskeln der Gesammtphosphor (A Tab. II) zunimmt, der Phosphor des wässerigen Extractes hingegen unverändert bleibt, so wächst natürlich die Differenz A-B (oder E Tab. II), d. h. der wasserunlösliche Phosphor zeigt eine Zunahme, ohne dass der wasserlösliche Phosphor vermindert erscheint. Vielleicht hängt die erwähnte Zunahme mit der Thatsache zusammen, dass aus den zerstörten organischen Substanzen auch Phosphate der Erdalkalien gebildet werden, oder dass der Phosphor, welcher dem Muskel durch das Blut zugeführt wird, bei der Regeneration seiner Substanz in wasserunlöslichen organischen Verbindungen - Lecithin oder Nuclein? - aufgestapelt wird.

Ich habe weiter gesucht, ob in den ruhenden und ermüdeten Hundemuskeln Bernsteinsäure vorkommt. Die 
hierbei angewandte Methode war die von Siegfried ${ }^{1}$ ) angegebene, welche sich bei einer Prüfung mittelst zugesetzter rciner Bernsteinsäure als sehr verlässlich erwies. Dieselbe besteht bekanntlich in Extraction des Muskels mit Alkohol, Behandlung des in Wasser gelösten Rückstandes der alkoholischen Flüssigkeit mit einem kleinen Ueberschusse von Barythydrat auf dem Wasserbade, Zersetzung der gebildeten Barytsalze mit wenig verdünnter Salzsäure in der Kälte und Ausäthern der Zersetzungsflüssigkeit. Es wurden so in zwei Versuchen an ruhenden und in zwei an ermüdeten Hunden erstens direkt alkoholische Auszüge, zweitens auch die durch Extraction mit salzsäurehaltigem Alkohol erhaltenen Auszüge auf das Vorhandensein von Bernsteinsäure untersucht.

Weder in ruhenden ${ }^{2}$ noch in erschöpften Muskeln konnte ich auf diese Weise Bernsteinsäure nachweisen.

Die hauptsächlichsten Resultate meiner Untersuchungen sind also, kurz gefasst, die folgenden:

1. Durch Muskelarbeit wird der organisch gebundene Phosphor des wässerigen Muskelextractes stark vermindert.

2. Dementsprechend werden die anorganischen Phosphate des wässerigen Muskelextractes vermehrt.

3. Der Nucleonphosphor im wässerigen Muskelextracte wird nur durch intensive Muskelanstrengung wesentlich vermindert.

4. Vor Allem wird der nicht dem Nucleon angehörige organische Phosphor des wässerigen Extractes durch Muskelarbeit sehr deutlich vermindert.

Die Versuche, über welche hier berichtet wurde, geben uns eine vorläufige Orientirung über das Verhalten der phosphor-

1) Diese Zeitschrift, Bd. XXI, S. 370.

2) Blumenthal, Virchow's Arch. f. Pathologie, Bd. CXXXVII, S. 539, konnte mit einer anderen Methode gleichfalls keine Bernsteinsäure in ruhenden Muskeln finden. 
haltigen Substanzen während der Arbeit. Sie geben uns aber keine Einsicht, welche Stoffe es neben dem Nucleon sind, die bei der Muskelthätigkeit verbraucht werden.

Weitere Untersuchungen in dieser Richtung behalte ich mir vor.

Zum Schluss erfülle ich die angenehme Pflicht, Herrn Prof. M. Siegfried für die gebotene Anregung und sehr freundliche Unterstützung bei dieser Arbeit und Herrn Dr. Burian für seine unermüdliche Unterstützung bei allen meinen Arbeiten im hiesigen Laboratorium meinen herzlichen Dank auszusprechen. 